\title{
Occurrence of valvar heart disease in acute rheumatic fever without evident carditis: colour- flow Doppler identification
}

\author{
Gordon M Folger Jr, Rachel Hajar, Andrej Robida, Hajar Ahmed Hajar
}

\begin{abstract}
Objective-To determine the frequency of occurrence of mitral and aortic valvar regurgitation in rheumatic children in whom there was no evidence of carditis acutely or at an earlier attack.

Design-Colour flow Doppler imaging was used in a non-randomised study of sequentially admitted children who met the criteria for acute rheumatic fever without clinically evident carditis and patients in whom the disease was quiescent after a previous attack of rheumatic fever. Two separate control groups were used for comparison of the echocardiographic findings, and a group of patients with confirmed rheumatic carditis was included for comparison of acute phase and antistreptococcal reactants.
\end{abstract}

Setting-A general hospital with the only paediatric inpatient department in Qatar.

Patients-From November 1988 to October 1990, 11 children were studied during the acute rheumatic period. In seven additional children the disease was quiescent when they were studied 18 to 36 months after a documented episode of acute rheumatic fever in which there was no evidence of carditis. The control patients were all studied during the same period.

Main outcome measure-Detection of mitral and aortic regurgitation in patients without clinical evidence of rheumatic carditis in the acute or quiescent stages of the disease.

Results-Mitral or mitral and aortic regurgitation was found in 10 of the 11 children studied in the acute rheumatic period. None had a murmur or other evidence of carditis. In all the cases studied the valvar insufficiency was mild. Four of the children studied late in the quiescent period had either aortic or mitral insufficiency by colour flow Doppler evaluation; two children who had previously had valvar insufficiency no longer showed this, and one child without positive findings in the acute phase remained without insufficiency. None of the non-rheumatic control subjects showed mitral or aortic regurgitation.

Conclusions-Colour flow Doppler imaging is a useful method of identifing subclinical mitral and aortic valvar disease at all stages of rheumatic fever when carditis cannot be otherwise detected and is a valuable addition to current diagnostic criteria.

It can be difficult to confirm the diagnosis of acute rheumatic fever when the only major manifestation is non-cardiac. Recurrences are common but with the patient's cooperation they can be prevented: when the heart is affected the prevention of a recurrence is vital.

We showed that Doppler echocardiography identified subclinical (that is, without auscultatory signs) degrees of valvar incompetence involving the mitral and aortic valves in children who had typical migratory polyarthritis as their only major rheumatic manifestation. ${ }^{1}$ The purpose of this report is to extend our previous findings by the use of colour flow Doppler echocardiographic studies in both the original group of patients and in newly occurring cases.

\section{Patients and methods}

Since our preliminary report that indicated a high incidence of unsuspected mitral and aortic valve disease in a small number of acute rheumatic fever patients without evidence of carditis, ${ }^{1}$ we have studied a consecutive series of new cases and patients from the earlier study by adding colour flow imaging to the study design. Four groups of children make up the patient population. Children in group 1 had confirmed acute rheumatic fever without clinical evidence of cardiac involvement: children evaluated since the end of the previous study made up group la and children from the original study were group 1b. Group 2 was made up of the new patients with obvious carditis who were compared with group la for indicators of acute rheumatic fever such as acute phase reactants. Group 2 was not included in the echocardiographic analysis because these children all had clinical evidence of mitral or aortic regurgitation when they entered the study. Group 3 includes children who were admitted to hospital with arthralgia or arthritis during the same period as those in group 1a and were subsequently shown not to have rheumatic fever. Patients in group 4 underwent diagnostic Doppler and colour flow echocardiography for other conditions suspected to be associated with cardiovascular problems.

The sole criterion for entrance into groups 1 to 3 of the study was suspicion by the admitting house officer of the possibility of acute rheumatic fever. In many instances this was a diagnosis by exclusion and with few exceptions was based primarily upon articular complaints 
and findings. A routine blood count, erythrocyte sedimentation rate, $\mathrm{C}$ reactive protein, antistreptolysin $\mathrm{O}$ titre, chest radiograph, and electrocardiogram were obtained at admission. To allow for night and weekend admissions complete cross sectional echocardiographic study including Doppler and Doppler colour flow imaging were performed within 48 hours of admission, and before any specific treatment for rheumatic fever was started.

The patients were all examined by at least two of us. At least one of us was also involved with the echocardiographic procedures. Except for group 4 patients, who were studied at random, the suspected diagnosis-rheumatic fever-was known to each of us at the time of echocardiographic study. In the initial clinical assessment and before echocardiographic evaluation, the newly presenting children in whom there was interobserver disagreement about the physical findings, principally the nature of any murmur detected, were accepted as having evidence of carditis and automatically assigned to group 2 .

All studies were performed with an Accuson 128. Colour flow imaging became available to us in November 1988. This was the starting date of the current study, which finished in October 1990.

The Doppler echocardiographic guidelines used were identical to those of the previous study. ${ }^{1}$ Briefly, regurgitant flow relative to the valve under investigation had to be holosystolic (mitral) or holodiastolic (aortic) with peak velocities for both exceeding $2.5 \mathrm{~m} / \mathrm{s}$; occasionally an envelope with terminal high velocity signals was difficult to obtain. All colour flow findings continued throughout the appropriate cardiac phase for that valve and clearly had to extend past the paravalvar region. Also, mosaic colour changes indicative of high velocity turbulence had to be seen.

\section{Results}

Every patient from the rheumatic groups ( 1 and 2) satisfied the major and minor aspects of the Jones criteria. Clinically the children in group 1 had only one major criterion (arthritis, chorea, or erythema marginatum) without any supportive clinical evidence of carditis; by contrast the group 2 patients all had auscultatory evidence of carditis or valvitis and thus had two major criteria. Several children from both groups had antistreptolysin $\mathrm{O}$ titres in the high normal range, a finding known to occur in roughly $20 \%$ of cases of acute rheumatic fever ${ }^{2}$; in our institution we perform no other streptococcal antibody investigations so that evidence of preceding streptococcal infection in these children was suspected but could not be confirmed.

\section{GROUP 1A}

Table 1 shows details of the 11 children in group 1a. This brings to a total of 21 , including those from the preliminary study, ${ }^{1}$ the children who make up our experience of patients with acute rheumatic fever without clinical evidence of carditis. Of these, nine presented with polyarthritis as their only major rheumatic symptom. One child had only a rash, considered to be that of erythema marginatum (patient 6). One child gave a history of chorea lasting more than one month and was having obvious choreiform movements when studied. Figures 1 and 2 show the colour flow Doppler images for this child.

No patient in group 1 showed any of the usually accepted clinical manifestations of active carditis; PR interval prolongation by itself, which was present in nearly $30 \%$ of these children, is generally considered non-specific and non diagnostic of rheumatic carditis. In addition to the absence of any murmurs even remotely suggestive of mitral or aortic regurgitation, indicators of carditis such as pericarditis, radiographic change in heart size, or tachycardia persisting longer than the febrile period were not found. Slightly less than half of these children had systolic murmurs of typically innocent quality and in the remainder no murmurs were heard. In no instance was there a history of previous rheumatic fever. All the patients, except one with chorea, were treated with aspirin as their only anti-inflammatory medication.

Table 1 Colour flow doppler imaging acute phase

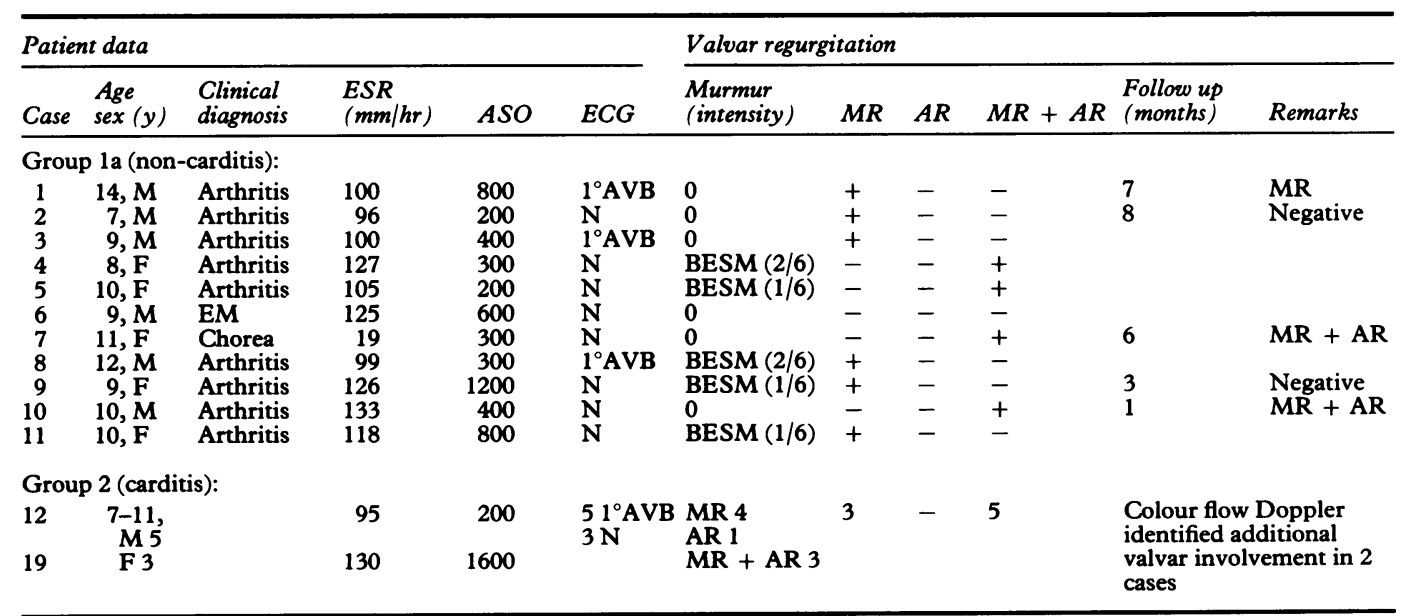

AR, aortic regurgitation; ASO, antistreptolysin O titre; AVB, atrioventricular block; BESM, basal ejection systolic murmur; $\mathrm{CH}$, chorea; EM, erythema marginatum; ESR, erythrocyte sedimentation rate; MR, mitral regurgitation; $N$, normal. 


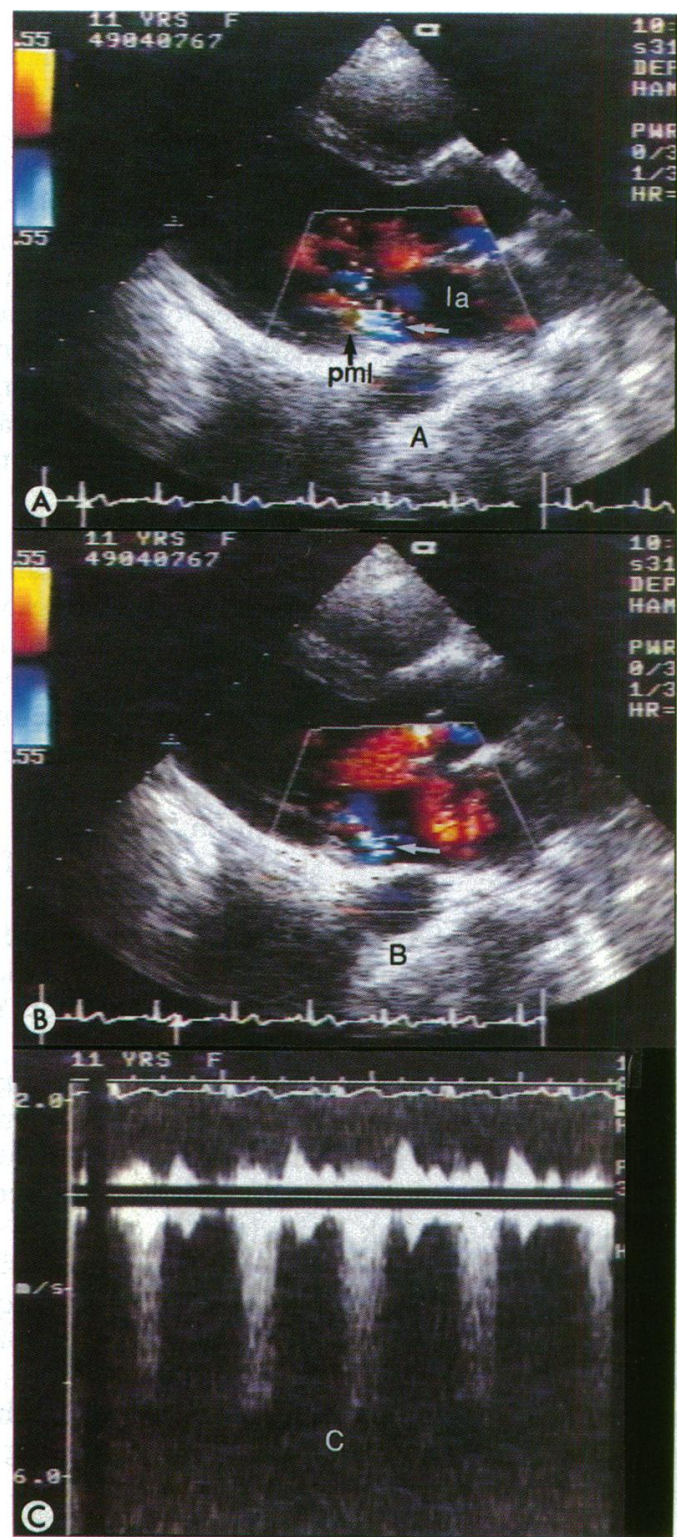

Figure 1 Colour flow Doppler imaging of mitral valve, parasternal long axis view in case 7. (A) Early systole. Note mosaic coloured flow signal (arrow) entering left atrium (1a). pml, posterior mitral valve leaflet. (B) Late systole. Holosystolic incompetence identified by the persistence of the regurgitant signal. (C) Continuous wave Doppler echocardiogram showing holosystolic high velocity flow away from transducer.

Colour flow Doppler imaging showed mitral regurgitation only in six children (cases $1,2,3$, $8,9,11)$ and mitral and aortic regurgitation in four (cases $4,5,7,10$ ). The only child without evidence of valvar incompetence was case 6 who had recurrent fever and a circinate rash, thought by many observers to be erythema marginatum. Both symptoms lasted for roughly one month; rheumatic fever in this patient has been neither confirmed nor ruled out. Ten of 11 children therefore, in this study group had evidence of either mitral or mitral and aortic regurgitation with no auscultatory findings.

GROUP 1B

Figure 3 shows that of the preliminary group of 10 subjects, ${ }^{1}$ seven had colour flow Doppler

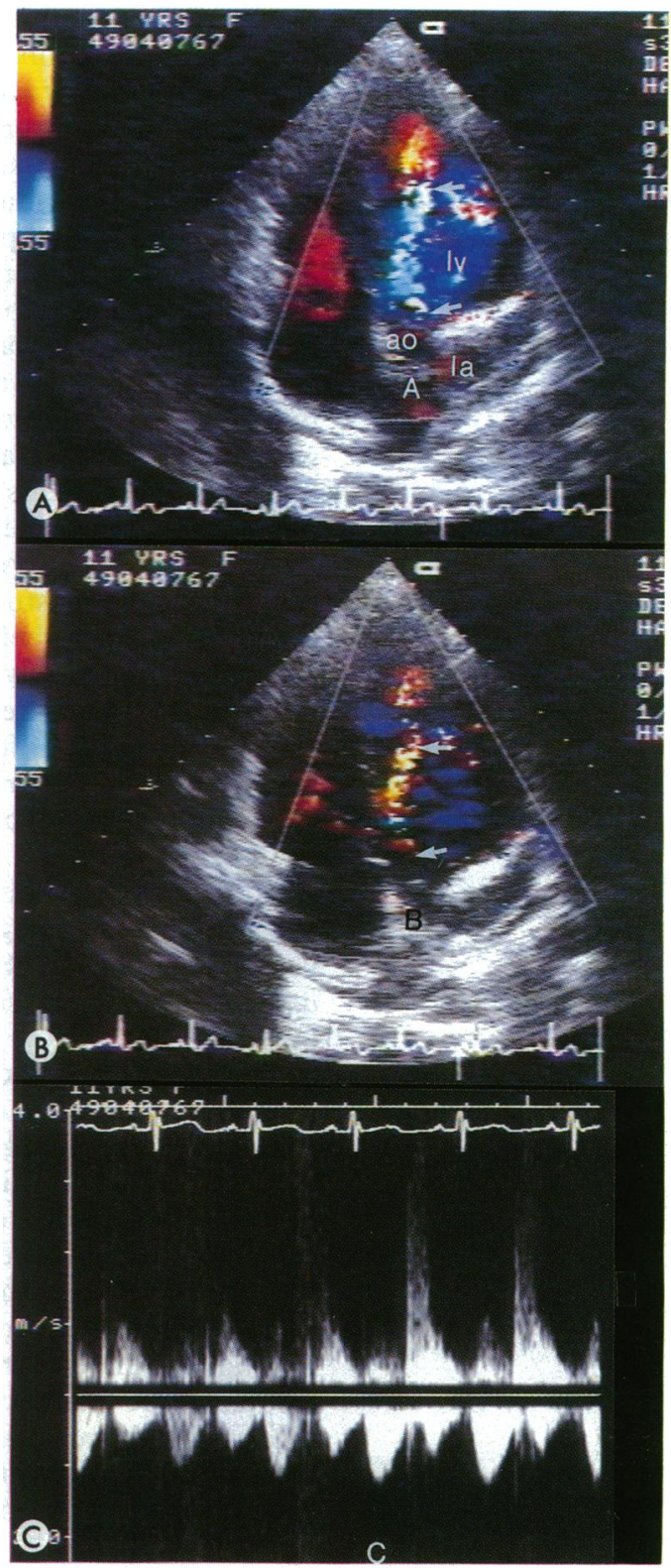

Figure 2 Colour flow Doppler imaging of aortic valve, four chamber view in case 7. (A) Early diastole. Note mosaic coloured flow signal. Arrows show the extent to which it extends below the aortic valve (ao). la, left atrium, lv, left ventricle. (B) Late diastole. Regurgitant signal persists identifying holodiastolic incompetence. Note that the appearance is now of minimal flow disturbance at the jet periphery with preservation of the central laminar core, effectively narrowing the jet width and possibly explaining the late high velocity signal drop out seen in panel C. (C) Continuous wave Doppler echocardiogram identifies high velocity regurgitant flow toward the transducer. The high velocity envelope
becomes attenuated as late diastole is approached.

analysis 18 months to three years after the acute episode. Of the six children who had positive Doppler echocardiographs in the acute period, four continued to show incompetence of at least one valve and two had become completely normal; none had developed murmurs of mitral or aortic regurgitation. One child who showed no positive findings in the acute period was not followed up. Two other patients not now available to the study, were positive by Doppler echocardiography early in the quiescent period. 
Group 1b

Doppler

Long-term follow up

Colour flow

echocardiogram

Doppler imaging

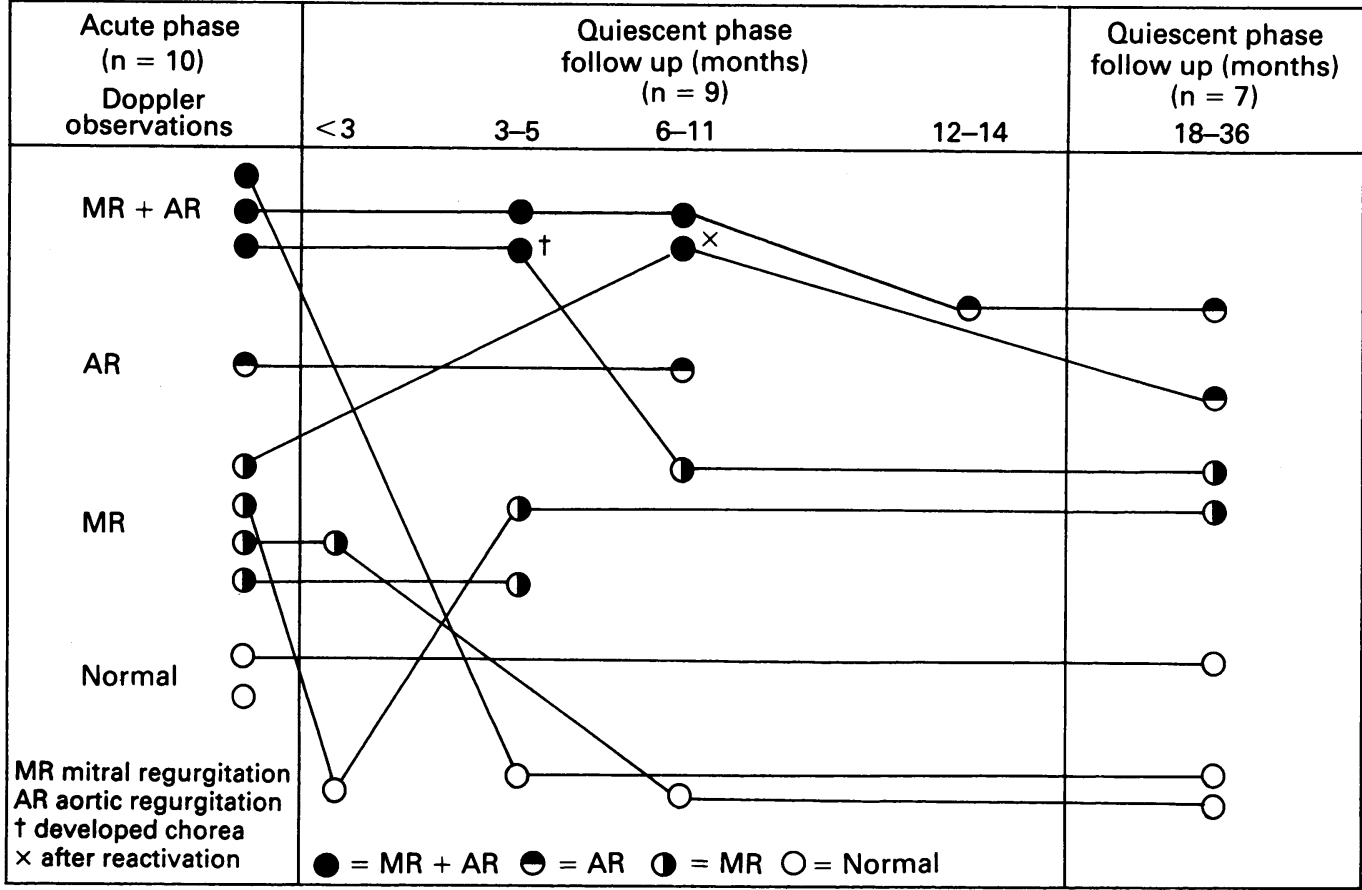

Figure 3 Long-term outcome of children with acute rheumatic fever without evident carditis. Four of the six children now undergoing follow up who showed Doppler echocardiographic evidence of valvar insufficiency in the acute period continued to have positive findings by colour flow Doppler 18 to 36 months later.

GROUP 2

Table 1 shows details of the eight children included in group 2 , who were all considered to have auscultatory evidence of carditis. The group was similar in age range and sex to those in group la. The ranges of their prolongations of $\mathbf{P} \mathbf{R}$ interval, acute phase reactants, and antistreptolysin $\mathrm{O}$ titres were also similar, as was their echocardiographically determined valvar involvement. It is noteworthy that colour flow Doppler analysis identified additional and clinically unsuspected valvar regurgitation in two of these children.

\section{CONTROL PATIENTS (GROUPS 3 AND 4)}

Table 2 shows details of groups 3 and 4 . The children included in group 3 served as controls because they were shown eventually not to have rheumatic fever. Each of these seven children, who ranged in age from four to 11 , had been admitted as possibly having acute rheumatic fever because of complaints and findings of arthritis or arthralgia. None of them showed evidence of regurgitation involving either the mitral or aortic valves. This finding was identical to that in the previous report. ${ }^{1}$ The occurrence of trivial flow into the left atrium in presystole that persisted briefly into early systole in one of these children was considered to be physiological.

The seven children aged four and a half to 16 who make up group 4 were studied during their admission for indications not related to rheumatic fever. None of them showed mitral or aortic incompetence.

\section{Discussion}

The present study confirmed our preliminary findings that the addition of Doppler echocardiography gave useful diagnostic information. ${ }^{1}$ We also found that the addition of colour flow

Table 2 Details of control patients

\begin{tabular}{llll}
\hline Group & Indication for study (n) & ESR (mm/hr)/ASO (units) & $\begin{array}{l}\text { Colour flow } \\
\text { Doppler }\end{array}$ \\
\hline $\begin{array}{l}\text { Group 3 } \\
\text { (age range 4 - 11) (F3 M4) }\end{array}$ & Tendinitis (1) & 0 \\
& Reactive polyarthritis (2) & $50 / 200$ & 0 \\
& Non-specific arthralgia (1) & $25 /<200$ & Phys MR \\
& Monarticular arthritis (1) & $37 /<200$ & 0 \\
& Anaphylactoid purpura (2) & $20 /<200$ & 0 \\
& & $50 / N P$ & 0 \\
Group 4 & & & 0 \\
(age range 4.5-16) (F4 M3) & Cardiac transplant (1) & & 0 \\
& Conduction abnormality (2) & NP & 0 \\
& Cbesity (1) & & TR (2) \\
\hline
\end{tabular}

NP, not performed; Phỳs MR, physiologic mitral regurgitation; PR, pulmonary regurgitation; TR, tricuspid regurgitation. 
imaging identified the site of the valve regurgitation and was especially useful in the small number of children in whom a complete spectral envelope could not be obtained (fig 2). This small study group represents a considerable experience of acute rheumatic fever. We are aware of no other study which Doppler echocardiography and Doppler colour flow mapping were used to detect valvar regurgitation in children without clinical evidence of carditis. Both methods detected regurgitation in a high proportion of patients. Indeed, the combined findings of 18 out of 21 patients studied who had mitral, or aortic regurgitation, or both, in the acute period is noteworthy. These findings accord with those of other groups who used different methods of detection. $^{34}$

Several groups have found mitral regurgitation by Doppler and colour flow imaging in normal children. ${ }^{5-7}$ From the descriptions of these it is apparent that the findings are usually those of abbreviated regurgitant flow into the left atrium, which we have also found. This occurs early in systole, occasionally starting in presystole, and seems to be a physiological event seen more commonly in older people. If our guidelines for mitral regurgitation were strictly followed overdiagnosis of organic mitral regurgitation is highly unlikely. Recently published reports ${ }^{5-7}$ and our own experience strongly suggest that non-organic (functional) mitral regurgitation does not produce holosystolic flow by Doppler echocardiography or colour flow imaging techniques. To our knowledge aortic regurgitation is not a normal finding.

These results have several implications for the diagnosis and management of patients with rheumatic fever. We believe that the colour flow Doppler findings should be added to the existing Jones criteria for diagnosis of acute rheumatic fever, ${ }^{8}$ especially because of the difficulty encountered when the Jones criteria are not completely satisfied. Because under the colour flow Doppler conditions described here these regurgitant flow patterns are not present in normal children and young adults, ${ }^{5-7}$ their presence when carditis is not clinically apparent should be of considerable value in the diagnosis of rheumatic fever. None of the control patients showed evidence of organic mitral or aortic regurgitation. As is usual, there was occasional tricuspid and pulmonary valvar regurgitation $^{5-7}$ in some of the controls as well as in several of the patients with rheumatic fever.

Some view the natural history of rheumatic fever when there is no clinical evidence of carditis as quite different and more favourable than when carditis is obvious. ${ }^{910}$ The term mimetic ${ }^{11}$ has been applied to each of these events suggesting reduced likelihood of valvar heart disease with recurrences of rheumatic fever when no carditis was obvious during the initial or subsequent bouts. The current findings however, indicate otherwise. They show that an appreciable number of these valves have been rendered incompetent regardless of the trivial nature of the findings, and many were still incompetent at follow up examination. To be more specific, these patients have valvar heart disease as a result of carditis that was not evident by the usual clinical means and this discovery is relevant to the perception of the natural history of pure rheumatic polyarthritis. Acute rheumatic fever without carditis is obviously not the benign entity that others have indicated, because it is nearly always accompanied by subclinical carditis, and the future of these patients is less certain than has been supposed. $^{9-11}$ This also applies to chorea without evident carditis.

Anatomical deformity aside, it may be that these valves become more vulnerable ${ }^{12}$ to damage by a recurrence of infection. This possibility must be considered before measures to prevent rheumatic damage are relaxed.

We believe that the addition of colour flow Doppler echocardiography to the Jones criteria for acute rheumatic fever would improve the identification of patients with mitral or aortic valve disease who require lifelong prophylaxis against rheumatic fever but who have no clinical evidence of cardiac involvement.

1 Folger GM Jr, Hajar R. Doppler echocardiographic findings of mitral and aortic valvular regurgitation in children manifesting only rheumatic arthritis. Am J Cardiol 1989; 63:1278-80.

2 Markowitz M, Kuttner AG. Rheumatic fever. Diagnosis management and prevention. Philadelphia: WB Saunders, management

3 Veasy LG, Wiedmeier SE, Orsmond GS, et al. Resurgence of acute rheumatic fever in the intermountain area of the of acute rheumatic fever in the intermountain
United States. $N$ Engl J Med 1987;316:421-7.

4 Steinfeld L, Ritter S, Rappaport H, Martinez E. Silent rheumatic mitral regurgitation unmasked by Doppler studies [abstract]. Circulation 1986;74 (suppl II):385.

5 Kostucki W, Vandenbossche J, Friart A, Englert M. Pulsed Doppler regurgitant flow patterns of normal valves. $A m J$ Cardiol 1986;58:309-13.

6 Yoshida K, Yoshikawa J, Shakudo M, et al. Color Doppler evaluation of valvular regurgitation in normal subjects. Circulation 1988;78:840-7.

7 Berger M, Hecht SR, Van Tosh A, Lingam U. Pulsed and continuous wave Doppler echocardiographic assessment of valvular regurgitation in normal subjects. $J$ Am Coll Cardiol 1989;13:1540-5.

8 Ad hoc committee to revise the Jones criteria (modified) of the council on rheumatic fever and congenital heart disease of the American Heart Association (1965). Jones disease of the American Heart Association (1965). Jones
criteria (revised) for guidance in the diagnosis of criteria (revised) for guidance in the
rheumatic fever. Circulation 1984;69:203-8.

9 Wenger NK, Leonard R, Bivins B. Acute rheumatic fever without carditis: a reappraisal. Am J Cardiol 1966;17: 142-3.

10 Committee on rheumatic fever and bacterial endocarditis of the Council on Cardiovascular Disease in the Young of the American Heart Association. Prevention of rheumatic fever. Circulation 1977;55:223-6.

11 Feinstein AR, Spagnuolo M. Mimetic features of rheumatic fever recurrences. N Engl J Med 1960;262:533-7.

12 Raizada V, Williams RC, Chopra P, et al. Tissue distribution of lymphocytes in reheumatic heart valves as defined by monoclonal anti-T cell antibodies. Am J Med 1983; 74:90-6. 